\title{
Neumonía intersticial linfocítica asociada a lupus eritematoso sistémico Una neumopatía infrecuente pero no inexistente
}

\author{
Lymphocytic interstitial pneumonia associated with \\ systemic lupus erythematosus. An uncommon, but not \\ inexistent condition
}

Martha Alejandra Casallas Rivera, MD. ${ }^{(1,3)}$; Alejandro González Muñoz, MD..$^{(1,2)}$; MARÍA JosÉ OSPINA PERDOMO, MD. ${ }^{(1,3)}$

\section{Resumen}

La neumonía intersticial linfocitaria es una entidad poco frecuente, constituida por una hiperplasia linfoide no neoplásica del tejido linfoideo intersticial pulmonar. Está asociada a fenómenos autoinmunes o infecciones virales, virus de la inmunodeficiencia humana o mononucleosis, así como al lupus eritematoso sistémico aunque es poco común. La sintomatología es variable: ttos seca, dolor torácico, disnea, y en menor frecuencia fiebre, artralgias y pérdida de peso.

Se presenta el caso de una mujer de 40 años de edad con antecedente de lupus eritematoso sistémico diagnosticado en 1998, quien presentó patología pulmonar compatible con neumonía intersticial linfocitaria confirmada por clínica, hallazgos radiológicos e histopatológicos; fue tratada con corticoide, ciclofosfamida, azatioprina e inmunoglobulina, pero debido al compromiso inmunológico ha desarrollado varias complicaciones infecciosas.

Palabras clave: neumonía, intersticial, hiperplasia, lupus eritematoso sistémico, micetoma, tuberculosis.

\begin{abstract}
Lymphocytic interstitial pneumonia is an uncommon condition characterized by non-neoplastic lymphoid hyperplasia of the interstitial lymphoid tissue of the lung. It is associated with autoimmune phenomena or viral infections, human immunodeficiency virus or mononucleosis, as well as systemic lupus erythematosus, although it is uncommon. The symptoms vary: dry cough, chest pain, dyspnea, and, less often, fever, arthralgia, and weight loss.

We present the case of a 40-year old female with a background of systemic lupus erythematosus diagnosed in 1998, who presented with pulmonary illness consistent with lymphocytic interstitial pneumonia, confirmed by clinical, radiologic, and histopathologic findings. She was treated with corticoids, cyclophosphamide, azathioprine, and immunoglobulin; however, due to her compromised immunologic system, she has developed several infectious complications.
\end{abstract}

Keywords: pneumonia, interstitial, hyperplasia, systemic lupus erythematosus, mycetoma, tuberculosis.

${ }^{(1)}$ Médico internista. Servicio de Medicina
Interna, Hospital Universitario de La Sa-
maritana. Bogotá, Colombia
${ }^{(2)}$ Estudiante de Medicina. Pontificia Uni-
versidad Javeriana. Bogotá, Colombia
${ }^{(3)}$ Residente de Medicina Interna. Univer-
sidad de La Sabana. Bogotá, Colombia.
Correspondencia: Martha Alejandra Casa-
llas Rivera, correo electrónico: m_aleja99@
hotmail.com
Recibido: 30/01/16. Aceptado: 20/01/16. 


\section{Introducción}

Las enfermedades pulmonares intersticiales son un grupo de patologías que afectan el intersticio pulmonar, y cuya característica común es la inflamación intersticial y la fibrosis (1).

La etiología de estas entidades es determinante para su clasificación fisiopatológica; entre ellas, las neumonías intersticiales idiopáticas son las más infrecuentes (2).

Bajo el término general de neumonía intersticial idiopática se incluyen la neumonía intersticial usual/ fibrosis pulmonar idiopática, la neumonía intersticial no específica, la neumonía intersticial descamativa, la bronquiolitis respiratoria asociada a enfermedad intersticial pulmonar, la neumonía organizada, la neumonía intersticial aguda y la neumonía intersticial linfocítica (3).

Esta última fue descrita por primera vez por Liebow y Carrington en 1966; es la menos común de estas entidades y ocurre como una reacción inflamatoria con infiltrado difuso policlonal de células linfoideas alrededor de la vía aérea, septos y tejido intersticial pulmonar (4]) células plasmáticas o histiocitos que infiltran el intersticio pulmonar y/o los espacios alveolares, probablemente a causa de estímulos externos o secundaria a enfermedades sistémicas asociadas (5).

Los infiltrados están dados fundamentalmente por linfocitos B y T, que forman centros germinales en los espacios peribronquiales hasta alcanzar el intersticio en estadios avanzados.

En las patologías asociadas a la neumonía intersticial linfocitaria se ha reportado, según la entidad, dominancia de celularidad citotóxica o ayudadora; por ejemplo, en pacientes con VIH se hallaron infiltrados de linfocitos citotóxicos CD8 (+) (6), mientras que en la hipogammaglobulinemia y en las patologías del sistema inmune se halló predominancia de linfocitos ayudadores CD4 (+) (7).

En cuanto a su epidemiología se desconoce mucha información debido a la falta de documentación y diagnóstico apropiado, siendo más frecuente en mujeres $(1: 2,75)$ con una edad promedio de 45 años (8).
La neumonía intersticial linfocitaria idiopática se observa en el $20 \%$ de los casos, mientras que un $39 \%$ de estos son ligados a enfermedades autoinmunes, con mayor frecuencia artritis reumatoidea seguida por síndrome de Sjögren en un 0,9\% y siendo muy rara en lupus eritematoso sistémico (LES), en cuyo caso se evidencia generalmente neumonía intersticial aguda (9); alrededor de 60 a $80 \%$ de los casos se asocia a disproteinemias, la mayoría hipergammaglobulinemia e infrecuentemente hipogammaglobulinemia, sin que necesariamente todas las personas afectadas por estas patologías desarrollen neumonía intersticial $(2,10,11)$. El síntoma más frecuente es la tos seca ( $70 \%$ de los casos) y en un menor porcentaje disnea, astenia, diaforesis, febrícula, pérdida de peso, artralgias y dolor torácico, ocasionalmente de características pleuríticas (8).

En la tomografía de alta resolución (TACAR) de tórax los hallazgos característicos incluyen áreas multifocales de vidrio deslustrado, generalmente bilateral y predominantemente en lóbulos inferiores, pequeños nódulos centrolobulillares de contornos mal definidos y quistes redondeados de paredes finas (figura 1) $(12,3)$, esto último asociado principalmente con enfermedad autoinmune (13).

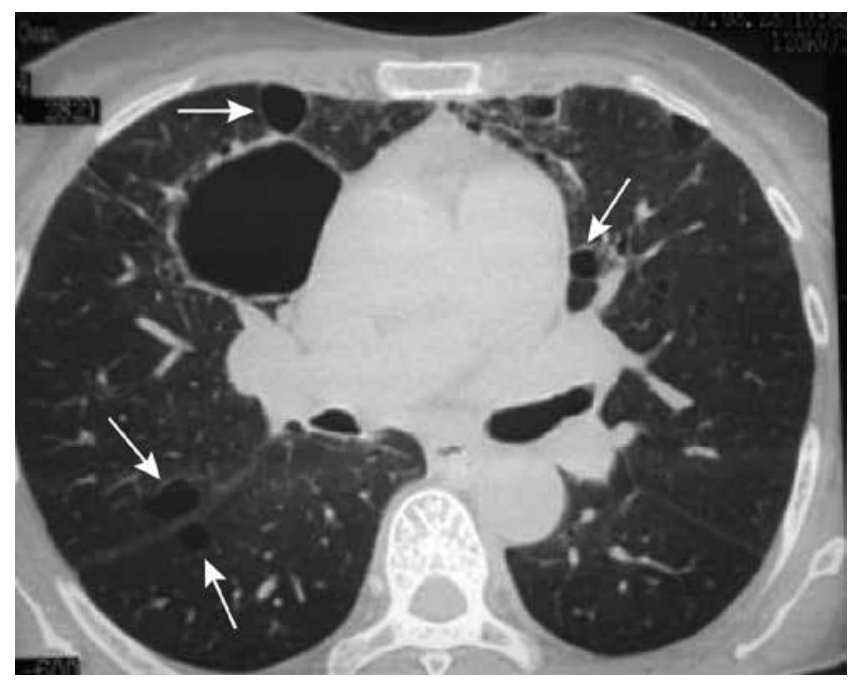

Figura 1. TACAR de tórax con presencia de múltiples quistes de pared delgada a nivel basal bilateral; patrón radiográfico de neumonía intersticial linfocitaria. 
En la biopsia pulmonar se esperaría encontrar las infiltraciones celulares del tejido; el problema del diagnóstico histológico radica en el tamaño de la muestra de la biopsia y en la heterogeneidad de las lesiones, de modo que la biopsia transbronquial convencional tiene un papel limitado, mientras que la biopsia pulmonar se hace de acuerdo con el estado del paciente y la experiencia del equipo médico, para establecer el pronóstico de la enfermedad.

Finalmente, el diagnóstico de esta entidad es avalado por los hallazgos de la escanografía de tórax de alta resolución TACAR de tórax y los hallazgos histológicos complementarios.

El tratamiento óptimo no está estandarizado y en la literatura solo se cuenta con recomendaciones de expertos. Se plantea el uso de corticoides con resolución completa del cuadro o estabilización de la sintomatología en algunos estudios (8), en tanto que en caso de mala respuesta o pobre pronóstico algunos autores describen el empleo de ciclosporina A sola o azatioprina y ciclofosfamida, ya sean individuales o con esteroides, las cuales han demostrado mejoría en pacientes con esta patología (14), para aquellos que tenían diagnóstico por alta sospecha y no por biopsia.

En pacientes con inmunodeficiencia común variable se ha sugerido adicionar inmunoglobulina IV mensual.

\section{Caso}

Paciente femenina de 40 años de edad, con diagnóstico de LES desde los 22 años de edad, quien entonces presentó fenómeno de Raynaud y sintomatología constitucional; posteriormente en 2007 tuvo cuadro caracterizado por tos sin expectoración y disnea progresiva no cuantificada, por lo cual fue estudiada por los servicios de Neumología y Reumatología quienes en TACAR de tórax encontraron patrón en "vidrio esmerilado" y áreas de consolidación multilobar derecha y espirometría con patrón restrictivo. Ordenaron biopsia transbronquial, la cual reportó parénquima pulmonar con tabiques interalveolares engrosados por infiltración linfocitaria de células maduras; áreas de cúmulos de histiocitos espumosos y ocasionales células gigantes multinucleadas. Con lo anterior se diagnosticó neumonía intersticial linfocitaria e iniciaron manejo con cuatro ciclos de ciclofosfamida; sin embargo, en febrero de 2008 se evidenció tuberculosis pulmonar por lo cual se suspendió el manejo con ciclofosfamida y se dio paso al manejo antituberculoso con criterios de curación, pero con fibrotórax derecho como secuela. Continuó en manejo crónico con azatropina (50 $\mathrm{mg} \mathrm{od}$ ), cloroquina (250 mg od), prednisolona (5 mg od), losartán (25 mg od), ácido acetilsalicílico (100 $\mathrm{mg}$ od), calcitriol $(0,25 \mu \mathrm{g}$ od), carbonato de calcio (600 mg od) y acetaminofén (1 g qid). En marzo de 2015, se registró dolor torácico tipo pleurítico, disnea MRC 3/4 (con previa de MRC 2), asociado a picos febriles, malestar general y cambios en el color y viscosidad de la expectoración por lo que recibió manejo antibiótico con ampicilina sulbactam; posteriormente, en mayo y junio, desarrolló un cuadro similar, en el que predominaron el malestar general y los picos febriles, así que fue hospitalizada para estudio. En nuevo TACAR de tórax se observó destrucción extensa del parénquima pulmonar derecho con grandes quistes de paredes delgadas versus bulas de aproximadamente $107 \mathrm{~mm}, 73 \mathrm{~mm}$ y $45 \mathrm{~mm}$ (figura 2) en el lóbulo superior, e imagen sugestiva de micetoma de $38 \mathrm{~mm}$ dentro del quiste ubicado en el

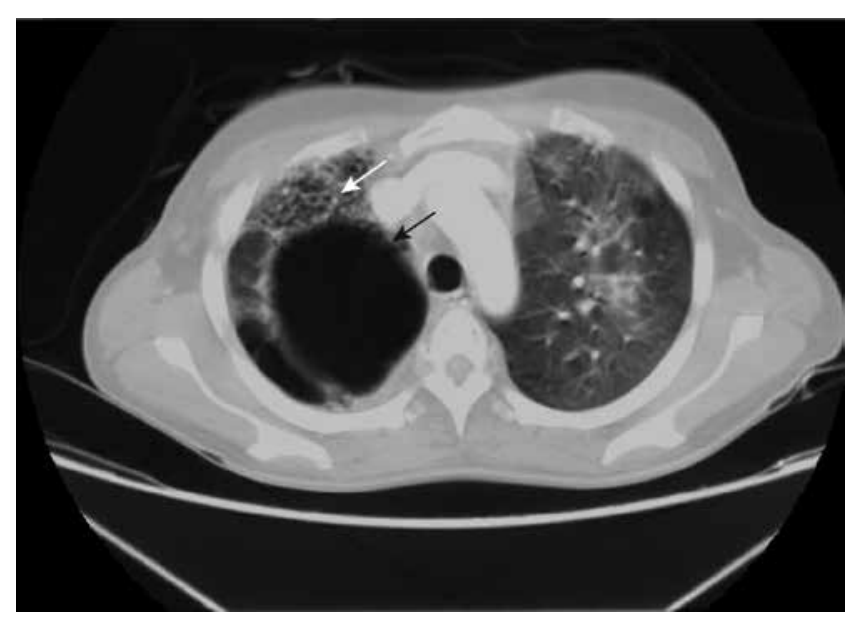

Figura 2. TACAR de tórax, que muestra múltiples quistes de pared delgada vs. bulas (flecha negra), patrón de "panal de abejas" (flecha blanca) en pulmón derecho y patrón en "vidrio esmerilado" en pulmón izquierdo. 
segmento posteromedial del lóbulo inferior derecho (figura 3). Adicionalmente, se observó "panalización" importante del parénquima pulmonar derecho con múltiples bronquiectasias. El hemitórax izquierdo mostró patrón en "vidrio esmerilado" difuso e infiltrados alveolares periféricos con predominio hacia segmentos posteroinferiores (figuras 3 y 4).

Por los hallazgos en el TACAR de tórax, la sintomatología, la fibrobroncoscopia (FBC) sugestiva de micosis, y adicionalmente por la aparición de Candida spp. y Klebsiella pneumoniae en cultivo de esputo, en junta médica se consideró iniciar caspofungina y piperacilina tazobactam, con lo cual se obtuvo mejoría parcial de los síntomas. Posteriormente con resultado histológico y microbiológico de $\mathrm{FBC}$ que reportó proceso inflamatorio agudo negativo para malignidad e infección, y por persistencia de fiebre al final de la terapia antimicrobiana, fue valorada por reumatólogo quien indicó manejo mensual con inmunoglobulinas por 6 meses, considerando actividad de la neumonía intersticial linfocitaria y ante no evidencia clínica ni paraclínica de actividad de su enfermedad inmunológica de base (tabla 1).

A pesar del manejo, persistió con síntomas y por tanto en nueva junta médica se decidió iniciar ciclofosfamida en ciclos mensuales.

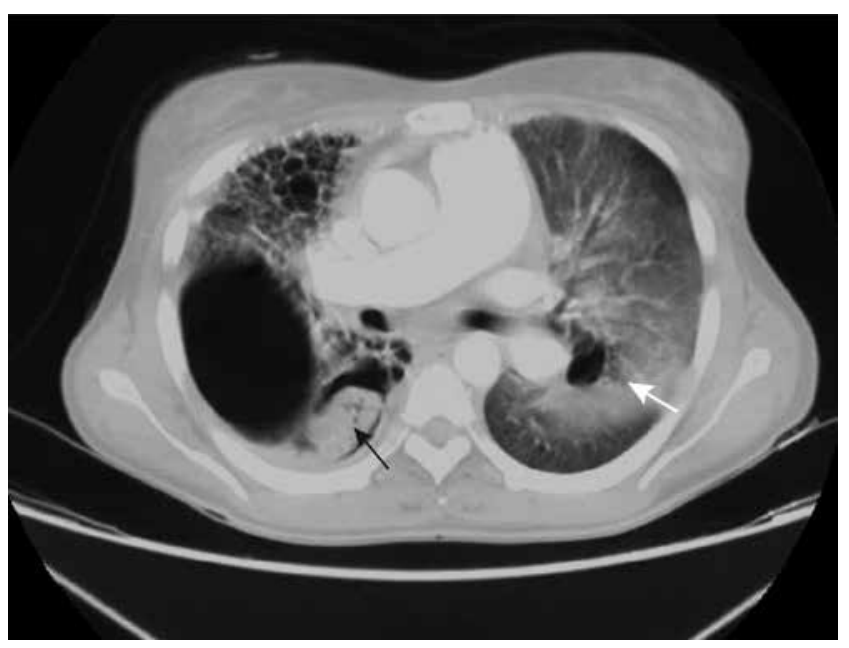

Figura 3. TACAR de tórax, en el que se evidencia micetoma (flecha negra) en región basal posteromedial derecha y patrón de vidrio esmerilado (flecha blanca) basal izquierdo.
Tabla 1. Perfil inmunológico del paciente.

Laboratorio
Complemento: normal
C3: 93,1
C4:23,9 Anti-DNA 1:40
Anticuerpos antinucleares (AAN) 1:320 patrón moteado
Anticuerpos extractables positivo:
Sm: $34,2 \quad$ SSA-Ro: 140,93

El día 28 de agosto de 2015, durante su manejo intrahospitalario, sufrió falla ventilatoria sin respuesta a maniobras de reanimación, y falleció.

\section{Discusión}

La neumonía intersticial linfocitaria es una entidad rara en nuestro medio y su asociación con LES es aún más infrecuente, enfrentándonos a la poca experiencia con la que se cuenta para su manejo.

Su etiología aun no es clara, sin embargo existe asociación con procesos donde hay producción de autoanticuerpos en el contexto de enfermedades

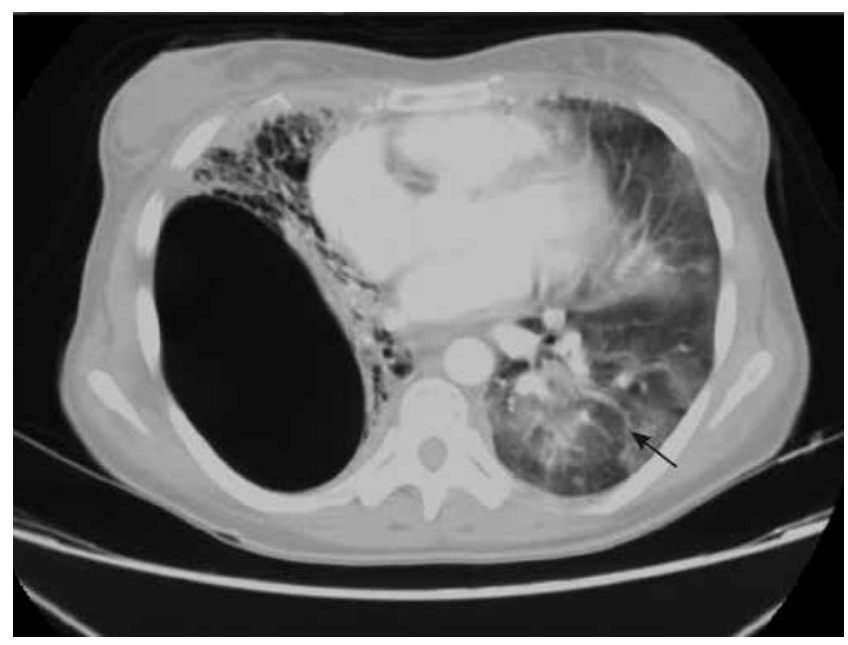

Figura 4. TACAR de tórax en el que se aprecian infiltrados alveolares (flecha roja) hacia la periferia en la base izquierda. 
reumáticas, principalmente síndrome de Sjögren y artritis reumatoide, y en menor medida con LES, miastenia gravis y anemia perniciosa, entre otros.

La enfermedad intersticial pulmonar crónica en LES es una complicación poco frecuente comparada con el resto de las enfermedades del tejido conectivo, con una prevalencia del 3 al 9\% (15), aunque en un tercio de los pacientes asintomáticos se aprecian leves alteraciones de la TACAR de tórax, los hallazgos más usuales son: vidrio deslustrado asociado a reticulación intralobulillar, líneas septales interlobulillares, bandas parenquimatosas y panalización $(15,16)$.

Los anticuerpos anti-Sm y anti SSA (Ro) se han relacionado con alteraciones en el sistema nervioso central y, en algunos casos, con neumopatía (17). Estudios previos han mostrado una asociación de anticuerpos anti-DNA y anti-Sm con trastornos respiratorios (17).

La hipertensión pulmonar consecutiva se puede explicar por hipoxemia y remodelación de la arquitectura pulmonar con cambios histológicos de fibrosis de la capa íntima en arterias pequeñas o de mediano calibre e hipertrofia concéntrica de las capas media e íntima de las arteriolas, necrosis fibrinoide y vasculitis, asociadas a engrosamiento de la capa íntima.

Se define daño fisiológico como capacidad vital forzada o difusión de monóxido de carbono menor a $70 \%$ del predicho como marcadores para iniciar tratamiento.

Este último se debe individualizar para el caso de las enfermedades reumáticas como LES, ya que en general las neumonitis asociadas a dicha enfermedad son menos agresivas que las idiopáticas. Se deberán descartar, además, infecciones mediante lavado broncoalveolar (BAL) o biopsia pulmonar. El tratamiento se basa en corticoides a altas dosis, y a veces es necesario adicionar otro inmunosupresor como ciclofosfamida, azatioprina o micofenolato (18); sin embargo sus resultados son variables. La media de supervivencia es 12 años. Se debe dar profilaxis para pneumocistosis en casos de dosis alta $(14,19)$.
En una serie de reportes de casos, uno con 18 participantes y otro con 13, se obtuvieron resultados similares incluso en pacientes que nunca recibieron tratamiento: en el 52\% mejoría y recuperación completa, en el 11\% estabilidad con persistencia del cuadro y en el $37 \%$ muerte por fibrosis pulmonar severa con una supervivencia media de 20 meses $(20,21)$.

\section{Conflicto de intereses}

Las autores declaran no tener conflicto de interés.

\section{Financiación}

Esta publicación fue financiada por los autores.

\section{Bibliografía}

1. Johkoh T, Müller NL, Pickford HA et al. Lymphocytic interstitial pneumonia: thin-section CT findings in 22 patients. Radiology. 1999;212(2):567-72.

2. Swigris JJ, Berry GJ, Raffin TA, Kuschner WG. Lymphoid interstitiael pneumonia: a narrative review. Chest. 2002;122:2150-64.

3. Franquet T, Giménez A. Neumonías intersticiales idiopáticas. Radiología. 2012; 54(6):479-89.

4. Davies CW, Juniper MC, Gray W, et al. Lymphoid interstitial pneumonitis associated with common variable hypogammaglobulinemia trated with cyclosporine A. Thorax. 2000;55:88-90.

5. Liebow AA, Carrington CB. Diffuse pulmonary lymphoreticular infiltrations associated with dysproteinemia. Med Clin North Am. 1973;57:809-43.

6. Travis WD, Fox CH, Devaney KO, et al. Lymphoid pneumonitis in 50 adult patients infected with the human immunodeficiency virus: lymphocytic interstitial pneumonitis versus nonspecific interstitial pneumonitis. Hum Pathol. 1992;23:529-41.

7. Church JA, Isaacs H, Saxon A, Keens TG, Richards W. Lymphoid interstitial pneumonitis and hypogammaglobulinemia in children. Am Rev Respir Dis. 1981;124:491-6.

8. Cha SI, Fessler MB, Cool CD, Schwarz MI, Brown KK. Lymphoid interstitial pneumonia: clinical features, associations and prognosis. Eur Respir J. 2006;28:364-9.

9. Gómez L. Enfermedades intersticiales en las conectivopatías. Rev Patol Respir. 2014;17(Supl.1):S53-S55.

10. Strimlan CV, Rosenow EC, Weiland LH, Brown LR. Lymphocytic interstitial pneumonitis. Review of 13 cases. Ann Intern Med. 1978;88:616-21.

11. Koss MN, Hochholzer L, Langloss JM, Wehunt WD, Lazarus AA. Lymphoid interstitial pneumonia: clinicopathological 
and immunopathological findings in 18 cases. Pathology.1987;19:178-85.

12. Mueller-Mang C, Grosse C, Schmid K, Stiebellehner L, Bankier AA. What every radiologist should know about idiopathic interstitial pneumonias. Radiographics. 2007;27:595-615.

13. Zamora AC, Collard HR, Ryu JH, et al. HRCT appearance predicts the underlying etiology of lymphocytic interstitial pneumonia (abstract). San Francisco, CA. ATS, Mayo; 2007.

14. Kondoh y, Taniguchi H, Yokoi T, et al. Cyclosphosphamide and low-dose prednisolone in idiopathic pulmonary fibrosis and fibrosing nonspecific interstitial pneumonia. Eur Respir J. 2005:25:528.

15. Gómez L, Bonilla G. Manifestaciones pulmonares de las enfermedades del colágeno. Arch Bronconeumol. 2013;49(6):249-60.

16. Ysamat R, Benito YA, Espejo PS, Blanco NM, Roldan MR.
La patología pulmonar asociada a las enfermedades del tejido conectivo. Radiología. 2013;55(2):107-17.

17. Zambrano GJA et al. Manifestaciones pulmonares en lupus eritematoso generalizado. Rev Med Hosp Gen Mex. 2004;67(1):41-9.

18. Koss MN, Hochholzer L, Langloss JM, et al. Lymphoid interstitial pneumonia: clinicopathological and immunopathological findings in 18 cases. Pathology. 1987;19:178.

19. Nanki N, Fujita J, Yamaji Y, et al. Nonspecific interstitial pneumonia/fibrosis completely recovered by adding cyclophosphamide to corticosteroids. Intern Med 2002:41:867.

20. Peralta G, Villagomez R, Bosio M, et al. Neumonía intesticial linfoide asociada a inmunodeficiencia común variable. MEDICINA (Buenos aires). 2011;71;462-4.

21. Strimlam CV, Rosenow EC, Weiland, LH, et al. Lymphocytic interstitial pneumonitis: A review of 13 cases. Ann Intern Med. 1978;68:616. 